\title{
A LOWER BOUND FOR THE NUMBER OF EDGES IN A GRAPH CONTAINING NO TWO CYCLES OF THE SAME LENGTH
}

\author{
Chunhui Lai * \\ Dept. of Math., Zhangzhou Teachers College, \\ Zhangzhou, Fujian 363000, P. R. of CHINA. \\ zjlaichu@public.zzptt.fj.cn
}

Submitted: November 3, 2000; Accepted: October 20, 2001.

MR Subject Classifications: 05C38, 05C35

Key words: graph, cycle, number of edges

\begin{abstract}
In 1975, P. Erdös proposed the problem of determining the maximum number $f(n)$ of edges in a graph of $n$ vertices in which any two cycles are of different lengths. In this paper, it is proved that

$$
f(n) \geq n+32 t-1
$$

for $t=27720 r+169(r \geq 1)$ and $n \geq \frac{6911}{16} t^{2}+\frac{514441}{8} t-\frac{3309665}{16}$. Consequently, $\liminf _{n \rightarrow \infty} \frac{f(n)-n}{\sqrt{n}} \geq \sqrt{2+\frac{2562}{6911}}$.
\end{abstract}

\section{Introduction}

Let $f(n)$ be the maximum number of edges in a graph on $n$ vertices in which no two cycles have the same length. In 1975, Erdös raised the problem of determining $f(n)$ (see [1], p.247, Problem 11). Shi[2] proved that

$$
f(n) \geq n+[(\sqrt{8 n-23}+1) / 2]
$$

for $n \geq 3$. Lai $[3,4,5,6]$ proved that for $n \geq(1381 / 9) t^{2}+(26 / 45) t+98 / 45, t=360 q+7$,

$$
f(n) \geq n+19 t-1,
$$

*Project Supported by NSF of Fujian(A96026), Science and Technology Project of Fujian(K20105) and Fujian Provincial Training Foundation for "Bai-Quan-Wan Talents Engineering". 
and for $n \geq e^{2 m}(2 m+3) / 4$,

$$
f(n)<n-2+\sqrt{n \ln (4 n /(2 m+3))+2 n}+\log _{2}(n+6) .
$$

Boros, Caro, Füredi and Yuster[7] proved that

$$
f(n) \leq n+1.98 \sqrt{n}(1+o(1))
$$

Let $v(G)$ denote the number of vertices, and $\epsilon(G)$ denote the number of edges. In this paper, we construct a graph $G$ having no two cycles with the same length which leads to the following result.

Theorem. Let $t=27720 r+169(r \geq 1)$, then

$$
f(n) \geq n+32 t-1
$$

for $n \geq \frac{6911}{16} t^{2}+\frac{514441}{8} t-\frac{3309665}{16}$.

\section{Proof of Theorem}

Proof. Let $t=27720 r+169, r \geq 1, n_{t}=\frac{6911}{16} t^{2}+\frac{514441}{8} t-\frac{3309665}{16}, n \geq n_{t}$. We shall show that there exists a graph $G$ on $n$ vertices with $n+32 t-1$ edges such that all cycles in $G$ have distinct lengths.

Now we construct the graph $G$ which consists of a number of subgraphs: $B_{i},(0 \leq$ $i \leq 21 t+\frac{7 t+1}{8}-58,22 t-798 \leq i \leq 22 t+64,23 t-734 \leq i \leq 23 t+267,24 t-531 \leq$ $i \leq 24 t+57,25 t-741 \leq i \leq 25 t+58,26 t-740 \leq i \leq 26 t+57,27 t-741 \leq i \leq$ $27 t+57,28 t-741 \leq i \leq 28 t+52,29 t-746 \leq i \leq 29 t+60,30 t-738 \leq i \leq 30 t+60$, and $31 t-738 \leq i \leq 31 t+799)$.

Now we define these $B_{i}$ 's. These subgraphs all have a common vertex $x$, otherwise their vertex sets are pairwise disjoint.

For $\frac{7 t+1}{8} \leq i \leq t-742$, let the subgraph $B_{19 t+2 i+1}$ consist of a cycle

$$
C_{19 t+2 i+1}=x x_{i}^{1} x_{i}^{2} \ldots x_{i}^{144 t+13 i+1463} x
$$

and eleven paths sharing a common vertex $x$, the other end vertices are on the cycle $C_{19 t+2 i+1}$ :

$$
\begin{aligned}
& x x_{i, 1}^{1} x_{i, 1}^{2} \ldots x_{i, 1}^{(11 t-1) / 2} x_{i}^{(31 t-115) / 2+i} \\
& x x_{i, 2}^{1} x_{i, 2}^{2} \ldots x_{i, 2}^{(13 t-1) / 2} x_{i}^{(51 t-103) / 2+2 i} \\
& x x_{i, 3}^{1} x_{i, 3}^{2} \ldots x_{i, 3}^{(13 t-1) / 2} x_{i}^{(71 t+315) / 2+3 i} \\
& x x_{i, 4}^{1} x_{i, 4}^{2} \ldots x_{i, 4}^{(15 t-1) / 2} x_{i}^{(91 t+313) / 2+4 i} \\
& x x_{i, 5}^{1} x_{i, 5}^{2} \ldots x_{i, 5}^{(15 t-1) / 2} x_{i}^{(111 t+313) / 2+5 i}
\end{aligned}
$$




$$
\begin{gathered}
x x_{i, 6}^{1} x_{i, 6}^{2} \ldots x_{i, 6}^{(17 t-1) / 2} x_{i}^{(131 t+311) / 2+6 i} \\
x x_{i, 7}^{1} x_{i, 7}^{2} \ldots x_{i, 7}^{(17 t-1) / 2} x_{i}^{(151 t+309) / 2+7 i} \\
x x_{i, 8}^{1} x_{i, 8}^{2} \ldots x_{i, 8}^{(19 t-1) / 2} x_{i}^{(171 t+297) / 2+8 i} \\
x x_{i, 9}^{1} x_{i, 9}^{2} \ldots x_{i, 9}^{(19 t-1) / 2} x_{i}^{(191 t+301) / 2+9 i} \\
x x_{i, 10}^{1} x_{i, 10}^{2} \ldots x_{i, 10}^{(21 t-1) / 2} x_{i}^{(211 t+305) / 2+10 i} \\
x x_{i, 11}^{1} x_{i, 11}^{2} \ldots x_{i, 11}^{(t-571) / 2} x_{i}^{(251 t+2357) / 2+11 i} .
\end{gathered}
$$

From the construction, we notice that $B_{19 t+2 i+1}$ contains exactly seventy-eight cycles of lengths:

$$
\begin{array}{llll}
21 t+i-57, & 22 t+i+7, & 23 t+i+210, & 24 t+i, \\
25 t+i+1, & 26 t+i, & 27 t+i, & 28 t+i-5, \\
29 t+i+3, & 30 t+i+3, & 31 t+i+742, & 19 t+2 i+1, \\
32 t+2 i-51, & 32 t+2 i+216, & 34 t+2 i+209, & 34 t+2 i, \\
36 t+2 i, & 36 t+2 i-1, & 38 t+2 i-6, & 38 t+2 i-3, \\
40 t+2 i+5, & 40 t+2 i+744, & 49 t+3 i+1312, & 42 t+3 i+158, \\
43 t+3 i+215, & 44 t+3 i+209, & 45 t+3 i-1, & 46 t+3 i-1, \\
47 t+3 i-7, & 48 t+3 i-4, & 49 t+3 i-1, & 50 t+3 i+746, \\
58 t+4 i+1314, & 53 t+4 i+157, & 53 t+4 i+215, & 55 t+4 i+208, \\
55 t+4 i-2, & 57 t+4 i-7, & 57 t+4 i-5, & 59 t+4 i-2, \\
59 t+4 i+740, & 68 t+5 i+1316, & 63 t+5 i+157, & 64 t+5 i+214, \\
65 t+5 i+207, & 66 t+5 i-8, & 67 t+5 i-5, & 68 t+5 i-3, \\
69 t+5 i+739, & 77 t+6 i+1310, & 74 t+6 i+156, & 74 t+6 i+213, \\
76 t+6 i+201, & 76 t+6 i-6, & 78 t+6 i-3, & 78 t+6 i+738, \\
87 t+7 i+1309, & 84 t+7 i+155, & 85 t+7 i+207, & 86 t+7 i+203, \\
87 t+7 i-4, & 88 t+7 i+738, & 96 t+8 i+1308, & 95 t+8 i+149, \\
95 t+8 i+209, & 97 t+8 i+205, & 97 t+8 i+737, & 106 t+9 i+1308, \\
105 t+9 i+151, & 106 t+9 i+211, & 107 t+9 i+946, & 115 t+10 i+1307, \\
116 t+10 i+153, & 116 t+10 i+952, & 125 t+11 i+1516, & 126 t+11 i+894, \\
134 t+12 i+1522, & 144 t+13 i+1464 . & &
\end{array}
$$

Similarly, for $58 \leq i \leq \frac{7 t-7}{8}$, let the subgraph $B_{21 t+i-57}$ consist of a cycle

$$
x y_{i}^{1} y_{i}^{2} \ldots y_{i}^{126 t+11 i+893} x
$$

and ten paths

$$
\begin{aligned}
& x y_{i, 1}^{1} y_{i, 1}^{2} \ldots y_{i, 1}^{(11 t-1) / 2} y_{i}^{(31 t-115) / 2+i} \\
& x y_{i, 2}^{1} y_{i, 2}^{2} \ldots y_{i, 2}^{(13 t-1) / 2} y_{i}^{(51 t-103) / 2+2 i} \\
& x y_{i, 3}^{1} y_{i, 3}^{2} \ldots y_{i, 3}^{(13 t-1) / 2} y_{i}^{(71 t+315) / 2+3 i}
\end{aligned}
$$




$$
\begin{gathered}
x y_{i, 4}^{1} y_{i, 4}^{2} \ldots y_{i, 4}^{(15 t-1) / 2} y_{i}^{(91 t+313) / 2+4 i} \\
x y_{i, 5}^{1} y_{i, 5}^{2} \ldots y_{i, 5}^{(15 t-1) / 2} y_{i}^{(111 t+313) / 2+5 i} \\
x y_{i, 6}^{1} y_{i, 6}^{2} \ldots y_{i, 6}^{(17 t-1) / 2} y_{i}^{(131 t+311) / 2+6 i} \\
x y_{i, 7}^{1} y_{i, 7}^{2} \ldots y_{i, 7}^{(17 t-1) / 2} y_{i}^{(151 t+309) / 2+7 i} \\
x y_{i, 8}^{1} y_{i, 8}^{2} \ldots y_{i, 8}^{(19 t-1) / 2} y_{i}^{(171 t+297) / 2+8 i} \\
x y_{i, 9}^{1} y_{i, 9}^{2} \ldots y_{i, 9}^{(19 t-1) / 2} y_{i}^{(191 t+301) / 2+9 i} \\
x y_{i, 10}^{1} y_{i, 10}^{2} \ldots y_{i, 10}^{(21 t-1) / 2} y_{i}^{(211 t+305) / 2+10 i}
\end{gathered}
$$

Based on the construction, $B_{21 t+i-57}$ contains exactly sixty-six cycles of lengths:

$$
\begin{array}{llll}
21 t+i-57, & 22 t+i+7, & 23 t+i+210, & 24 t+i, \\
25 t+i+1, & 26 t+i, & 27 t+i, & 28 t+i-5, \\
29 t+i+3, & 30 t+i+3, & 31 t+i+742, & 32 t+2 i-51, \\
32 t+2 i+216, & 34 t+2 i+209, & 34 t+2 i, & 36 t+2 i, \\
36 t+2 i-1, & 38 t+2 i-6, & 38 t+2 i-3, & 40 t+2 i+5, \\
40 t+2 i+744, & 42 t+3 i+158, & 43 t+3 i+215, & 44 t+3 i+209, \\
45 t+3 i-1, & 46 t+3 i-1, & 47 t+3 i-7, & 48 t+3 i-4, \\
49 t+3 i-1, & 50 t+3 i+746, & 53 t+4 i+157, & 53 t+4 i+215, \\
55 t+4 i+208, & 55 t+4 i-2, & 57 t+4 i-7, & 57 t+4 i-5, \\
59 t+4 i-2, & 59 t+4 i+740, & 63 t+5 i+157, & 64 t+5 i+214, \\
65 t+5 i+207, & 66 t+5 i-8, & 67 t+5 i-5, & 68 t+5 i-3, \\
69 t+5 i+739, & 74 t+6 i+156, & 74 t+6 i+213, & 76 t+6 i+201, \\
76 t+6 i-6, & 78 t+6 i-3, & 78 t+6 i+738, & 84 t+7 i+155, \\
85 t+7 i+207, & 86 t+7 i+203, & 87 t+7 i-4, & 88 t+7 i+738, \\
95 t+8 i+149, & 95 t+8 i+209, & 97 t+8 i+205, & 97 t+8 i+737, \\
105 t+9 i+151, & 106 t+9 i+211, & 107 t+9 i+946, & 116 t+10 i+153, \\
116 t+10 i+952, & 126 t+11 i+894 . & &
\end{array}
$$

$B_{0}$ is a path with an end vertex $x$ and length $n-n_{t}$. Other $B_{i}$ is simply a cycle of length $i$.

It is easy to see that

$$
\begin{aligned}
& v(G)=v\left(B_{0}\right)+\sum_{i=1}^{19 t+\frac{7 t+1}{4}}\left(v\left(B_{i}\right)-1\right)+\sum_{i=\frac{7 t+1}{8}}^{t-742}\left(v\left(B_{19 t+2 i+1}\right)-1\right) \\
& +\sum_{i=\frac{7 t+1}{8}}^{t-742}\left(v\left(B_{19 t+2 i+2}\right)-1\right)+\sum_{i=21 t-1481}^{21 t}\left(v\left(B_{i}\right)-1\right) \\
& +\sum_{i=58}^{\frac{7 t-7^{8}}{8}}\left(v\left(B_{21 t+i-57}\right)-1\right)+\sum_{i=22 t-798}^{22 t+64}\left(v\left(B_{i}\right)-1\right)+\sum_{i=23 t-734}^{23 t+267}\left(v\left(B_{i}\right)-1\right) \\
& +\sum_{i=24 t-531}^{24 t+57}\left(v\left(B_{i}\right)-1\right)+\sum_{i=25 t-741}^{25 t+58}\left(v\left(B_{i}\right)-1\right)+\sum_{i=26 t-740}^{26 t+57}\left(v\left(B_{i}\right)-1\right) \\
& +\sum_{i=27 t-741}^{27 t+57}\left(v\left(B_{i}\right)-1\right)+\sum_{i=28 t-741}^{28 t+52}\left(v\left(B_{i}\right)-1\right)+\sum_{i=29 t-746}^{29 t+60}\left(v\left(B_{i}\right)-1\right) \\
& +\sum_{i=30 t-738}^{30 t+60}\left(v\left(B_{i}\right)-1\right)+\sum_{i=31 t-738}^{31 t+799}\left(v\left(B_{i}\right)-1\right)
\end{aligned}
$$




$$
\begin{aligned}
& =n-n_{t}+1+\sum_{i=1}^{19 t+\frac{7 t+1}{4}}(i-1)+\sum_{i=\frac{7 t+1}{8}}^{t-742}(144 t+13 i+1463 \\
& +\frac{11 t-1}{2}+\frac{13 t-1}{2}+\frac{13 t-1}{2}+\frac{15 t-1}{2}+\frac{15 t-1^{8}}{2}+\frac{17 t-1}{2}+\frac{17 t-1}{2} \\
& \left.+\frac{19 t-1}{2}+\frac{19 t-1}{2}+\frac{21 t-1}{2}+\frac{t-571}{2}\right)+\sum_{i=\frac{7 t+1}{8}}^{t-742}(19 t+2 i+1) \\
& +\sum_{i=21 t-1481}^{21 t}(i-1)+\sum_{i=58}^{\frac{7 t-7}{8}}(126 t+11 i+893 \\
& +\frac{11 t-1}{2}+\frac{13 t-1}{2}+\frac{13 t-1}{2}+\frac{15 t-1}{2}+\frac{15 t-1}{2}+\frac{17 t-1}{2}+\frac{17 t-1}{2} \\
& \left.+\frac{19 t-1}{2}+\frac{19 t-1}{2}+\frac{21 t-1}{2}\right)+\sum_{i=22 t-798}^{2}(i-1) \\
& +\sum_{i=23 t-734}^{23 t+267}(i-1)+\sum_{i=24 t-531}^{24 t+57}(i-1)+\sum_{i=25 t-741}^{25 t+58}(i-1) \\
& +\sum_{i=26 t-740}^{26 t+57}(i-1)+\sum_{i=27 t-741}^{27 t+57}(i-1)+\sum_{i=28 t-741}^{28 t+52}(i-1) \\
& +\sum_{i=29 t-746}^{29 t+60}(i-1)+\sum_{i=30 t-738}^{30 t+60}(i-1)+\sum_{i=31 t-738}^{31 t+799}(i-1) \\
& =n-n_{t}+\frac{1}{16}\left(-3309665+1028882 t+6911 t^{2}\right) \\
& =n \text {. }
\end{aligned}
$$

Now we compute the number of edges of $G$

$$
\begin{aligned}
& \epsilon(G)=\epsilon\left(B_{0}\right)+\sum_{i=1}^{19 t+\frac{7 t+1}{4}} \epsilon\left(B_{i}\right)+\sum_{i=\frac{7 t+1}{8}}^{t-742} \epsilon\left(B_{19 t+2 i+1}\right) \\
& +\sum_{i=\frac{7 t+1}{8}}^{t-742} \epsilon\left(B_{19 t+2 i+2}\right)+\sum_{i=21 t-1481}^{21 t} \epsilon\left(B_{i}\right) \\
& +\sum_{i=58}^{\frac{7 t-7^{8}}{8}} \epsilon\left(B_{21 t+i-57}\right)+\sum_{i=22 t-798}^{22 t+64} \epsilon\left(B_{i}\right)+\sum_{i=23 t-734}^{23 t+267} \epsilon\left(B_{i}\right) \\
& +\sum_{i=24 t-531}^{24 t+57} \epsilon\left(B_{i}\right)+\sum_{i=25 t-741}^{25 t+58} \epsilon\left(B_{i}\right)+\sum_{i=26 t-740}^{26 t+57} \epsilon\left(B_{i}\right) \\
& +\sum_{i=27 t-741}^{27 t+57} \epsilon\left(B_{i}\right)+\sum_{i=28 t-741}^{28 t+52} \epsilon\left(B_{i}\right)+\sum_{i=29 t-746}^{29 t+60} \epsilon\left(B_{i}\right) \\
& +\sum_{i=30 t-738}^{30 t+60} \epsilon\left(B_{i}\right)+\sum_{i=31 t-738}^{31 t+799} \epsilon\left(B_{i}\right) \\
& =n-n_{t}+\sum_{i=1}^{19 t+\frac{7 t+1}{4}} i+\sum_{i=\frac{7 t+1}{8}}^{t-742}(144 t+13 i+1464 \\
& +\frac{11 t+1}{2}+\frac{13 t+1}{2}+\frac{13 t+1}{2}+\frac{15 t+1}{2}+\frac{15 t+1}{2}+\frac{17 t+1}{2}+\frac{17 t+1}{2} \\
& \left.+\frac{19 t+1}{2}+\frac{19 t+1}{2}+\frac{21 t+1}{2}+\frac{t-571+2}{2}\right)+\sum_{i=\frac{7 t+1}{8}}^{t}(19 t+2 i+2) \\
& +\sum_{i=21 t-1481}^{21 t} i+\sum_{i=58}^{\frac{7 t-7}{8}}(126 t+11 i+894 \\
& +\frac{11 t+1}{2}+\frac{13 t+1}{2}+\frac{13 t+1}{2}+\frac{15 t+1}{2}+\frac{15 t+1}{2}+\frac{17 t+1}{2}+\frac{17 t+1}{2} \\
& \left.+\frac{19 t+1}{2}+\frac{19 t+1}{2}+\frac{21 t+1}{2}\right)+\sum_{i=22 t-798}^{2} i \\
& +\sum_{i=23 t-734}^{23 t+267} i+\sum_{i=24 t-531}^{24 t+57} i+\sum_{i=25 t-741}^{25 t+58} i \\
& +\sum_{i=26 t-740}^{26 t+57} i+\sum_{i=27 t-741}^{27 t+57} i+\sum_{i=28 t-741}^{28 t+52} i \\
& +\sum_{i=29 t-746}^{299 t+60} i+\sum_{i=30 t-738}^{30 t+60} i+\sum_{i=31 t-738}^{31 t+799} i \\
& =n-n_{t}+\frac{1}{16}\left(-3309681+1029394 t+6911 t^{2}\right) \\
& =n+32 t-1 \text {. }
\end{aligned}
$$

Then $f(n) \geq n+32 t-1$, for $n \geq n_{t}$. This completes the proof of the theorem.

From the above theorem, we have

$$
\liminf _{n \rightarrow \infty} \frac{f(n)-n}{\sqrt{n}} \geq \sqrt{2+\frac{2562}{6911}}
$$

which is better than the previous bounds $\sqrt{2}$ (see [2]), $\sqrt{2+\frac{487}{1381}}$ (see [6]).

Combining this with Boros, Caro, Füredi and Yuster's upper bound, we have

$$
1.98 \geq \limsup _{n \rightarrow \infty} \frac{f(n)-n}{\sqrt{n}} \geq \liminf _{n \rightarrow \infty} \frac{f(n)-n}{\sqrt{n}} \geq 1.5397 .
$$




\section{Acknowledgment}

The author thanks Prof. Yair Caro and Raphael Yuster for sending reference [7]. The author also thanks Prof. Cheng Zhao for his advice.

\section{References}

[1] J.A. Bondy and U.S.R. Murty, Graph Theory with Applications (Macmillan, New York, 1976).

[2] Y. Shi, On maximum cycle-distributed graphs, Discrete Math. 71(1988) 57-71.

[3] Chunhui Lai, On the Erdös problem, J. Zhangzhou Teachers College(Natural Science Edition) 3(1)(1989) 55-59.

[4] Chunhui Lai, Upper bound and lower bound of $f(n)$, J. Zhangzhou Teachers College(Natural Science Edition) 4(1)(1990) 29,30-34.

[5] Chunhui Lai, On the size of graphs with all cycle having distinct length, Discrete Math. 122(1993) 363-364.

[6] Chunhui Lai, The edges in a graph in which no two cycles have the same length, J. Zhangzhou Teachers College (Natural Science Edlition) 8(4)(1994), 30-34.

[7] E. Boros, Y. Caro, Z. Füredi and R. Yuster, Covering non-uniform hypergraphs (submitted, 2000). 